Discrete Optimization

\title{
Allocation strategies in hub networks
}

\author{
Hande Yaman* \\ Bilkent University, Department of Industrial Engineering, Bilkent 06800, Ankara, Turkey
}

\section{A R T I C L E I N F O}

\section{Article history:}

Received 5 April 2010

Accepted 8 January 2011

Available online 15 January 2011

\section{Keywords:}

Location

Hub location

p-Hub median

Single allocation

Multiple allocation

\begin{abstract}
A B S T R A C T
In this paper, we study allocation strategies and their effects on total routing costs in hub networks. Given a set of nodes with pairwise traffic demands, the $p$-hub median problem is the problem of choosing $p$ nodes as hub locations and routing traffic through these hubs at minimum cost. This problem has two versions; in single allocation problems, each node can send and receive traffic through a single hub, whereas in multiple allocation problems, there is no such restriction and a node may send and receive its traffic through all $p$ hubs. This results in high fixed costs and complicated networks. In this study, we introduce the $r$-allocation $p$-hub median problem, where each node can be connected to at most $r$ hubs. This new problem generalizes the two versions of the $p$-hub median problem. We derive mixedinteger programming formulations for this problem and perform a computational study using wellknown datasets. For these datasets, we conclude that single allocation solutions are considerably more expensive than multiple allocation solutions, but significant savings can be achieved by allowing nodes to be allocated to two or three hubs rather than one. We also present models for variations of this problem with service quality considerations, flow thresholds, and non-stop service.
\end{abstract}

(C) 2011 Elsevier B.V. All rights reserved.

\section{Introduction}

Hubs are facilities that serve as switching points in telecommunications and transportation networks. Hub networks arise where there is traffic demand from many origin nodes to many destination nodes and connecting all origin-destination pairs by direct links is not practical and/or economical. Flows from many origins to many destinations are consolidated at hubs and routed together to benefit from economies of scale. The $p$-hub median problem can be defined as follows: given a set of nodes with pairwise traffic demands, choose $p$ nodes to locate hubs and route the traffic of all nodes through these hubs at minimum cost. It is often assumed that hubs are connected by a complete network, the routing cost between hubs is discounted at a factor $0<\alpha \leqslant 1$, and no direct connection exists between two nonhub nodes.

Recently, there have been studies on various extensions of this problem, where the basic assumptions about the structure of the network and the way the costs are discounted are relaxed. For instance, O'Kelly and Miller (1994), Nickel et al. (2001), Yoon and Current (2008), Calik et al. (2009), and Alumur et al. (2009) consider hub location problems where the hub network is not necessarily complete. Labbé and Yaman (2008), and Yaman (2008) consider star hub networks, whereas Contreras et al. (2010) study

\footnotetext{
* Tel.: +90 31229027 68; fax: +90 3122664054 .

E-mail address: hyaman@bilkent.edu.tr
}

a tree structure. Yaman (2009) studies the problem of designing hierarchical hub networks where the top-level hub network is complete and the second-level hub networks are stars. Campbell et al. (2005a,b) study the problem of locating a given number of hub arcs with discounted costs rather than locating hubs. Podnar et al. (2002) discount the transportation cost on a link if the flow on this link exceeds a threshold. O'Kelly and Bryan (1998), and Horner and O'Kelly (2001) relax the assumption of a fixed discount factor on hub-to-hub links and model economies of scale as a function of flow.

In extending the $p$-hub median problem, the focus has been primarily on hub-to-hub connections. In this study, we keep the assumptions concerning the hub network and focus on the allocation of nodes to hubs. In the literature, there are two versions of the $p$-hub median problem that differ in their allocation strategies. In the single allocation version, each node can send and receive traffic through exactly one hub node, whereas in the multiple allocation version, there is no such restriction; a node may be connected to all $p$ hubs. In both versions of the $p$-hub median problem, the total cost is equal to the routing cost. The fixed costs of connections between nonhub and hub nodes and among hub nodes are not considered. However, in reality, each connection requires the use of a capacity, in the form of a vehicle in a transportation application and in the form of fibers and technical equipments in a telecommunications application. Hence, a hub network where nodes are connected to almost all hubs by direct links may be difficult to justify economically. On the other hand, imposing the restriction of 
single allocation may result in a considerable increase in routing costs.

Networks where each node is connected to exactly one hub and networks where each node is connected to all $p$ hubs are two extremes. In practice, however, most hub networks do not belong to either of these cases. For instance, the airline networks, which are key examples of hub networks, are usually not single allocation networks. It is also hard to find examples of airline networks where each node is connected to every hub in the network unless the number of hubs is very small.

Motivated by the structure of hub networks that we encounter in practice and the fact that multiple allocation solutions are often significantly cheaper than single allocation solutions in terms of the total transportation cost but that they have a larger number of nonhub-hub connections, in this study we investigate the trade-off between transportation costs and the number of nonhub-hub connections and show that the issue of allocation deserves more attention when designing hub networks.

To avoid an excessive number of nonhub-hub connections, one can include the fixed costs in the objective function in addition to the routing costs, and/or impose lower bounds on the traffic on open links. Campbell (1994) proposes the $p$-hub median problem with flow thresholds and fixed costs for nonhub-hub connections. He presents a model for this problem and then focuses on the case with zero fixed costs. In this case, if the threshold is zero, the problem becomes a multiple allocation problem and if the threshold for a given node is equal to the total flow at that node, then the problem becomes a single allocation problem. Later, Yoon and Current (2008) propose a model that includes fixed costs as well as variable arc costs.

However, it is often difficult to quantify the relationship between fixed and variable costs. In order to avoid this difficulty and still limit the amount of fixed costs, we propose to restrict the number of connections between a node and a hub node by a fixed number. To this end, we define the uncapacitated r-allocation p-hub median problem (UrApHMP) as follows. Given a set of nodes with pairwise demands, choose $p$ hubs and allocate each node to at most $r$ hubs to minimize the total routing cost. This problem reduces to the multiple allocation problem for $r=p$ and it reduces to the single allocation problem for $r=1$. Hence the UrApHMP generalizes both versions of the $p$-hub median problem.

In this study, we present mixed-integer programming models for UrApHMP and unify the existing models for the multiple and single allocation $p$-hub median problems. These models for UrApHMP serve as the basis for modeling different problem variations. For instance, they can be trivially modified to model a more realistic version of the problem, where the upper bound on the number of connections for a node depends on the total traffic demand at that node. As another variation, we minimize the fixed costs of nonhub-hub connections subject to a constraint that imposes an upper bound on the total transportation cost. The aim here is to see the trade-off between the transportation cost and the fixed costs of nonhub-hub connections by solving this problem for different values of upper bounds on the transportation cost. The problem of minimizing the sum of fixed costs of nonhub-hub connections and the transportation cost subject to flow thresholds for nonhub-hub connections, studied by Campbell (1994), can also be easily modeled.

We also consider quality issues and incorporate path-length constraints and delivery time restrictions into UrApHMP. To the best of our knowledge, the multiple allocation $p$-hub median problem with delivery time restrictions has not been studied. The UrApHMP with delivery time restrictions has this problem as a special case and hence we give, as a byproduct, a model for the multiple allocation $p$-hub median problem with delivery time restrictions.
Finally, we present a maximization version of the problem where the demand from an origin to a destination is a function of the number of hubs traversed and non-stop service is allowed.

To motivate the study on the $r$-allocation $p$-hub median problem, we report the outcomes of an analysis for the CAB data with 25 nodes, $p=5$, and $\alpha=1,0.8$. This data is introduced in O'Kelly (1987) and is based on airline traffic between 25 US cities. First, we compare the minimum routing costs for single and multiple allocation solutions: The single allocation network is around $20 \%$ and $13.5 \%$ more expensive than the multiple allocation network for $\alpha=1$ and $\alpha=0.8$, respectively. When we study the allocations in the multiple allocation optimal solutions, we observe the following: When $\alpha=1$, each nonhub node is allocated to all five hubs in the solution, resulting in 100 nonhub-hub connections. For $\alpha=0.8$, out of 25 nodes, seven nodes are allocated to four hubs, eight nodes to three hubs, one node to two hubs, and the remaining nine nodes to a single hub. On average, a node is allocated to 2.52 hubs in this solution. When we solve the $r$-allocation $p$-hub median problem for $r=3$ and $r=2$, the routing costs for $r=3$ turned out to be almost the same as the routing costs for $r=p=5$. Hence, limiting the number of allocations to at most three does not cause a significant increase in the routing costs but it does result in a significant reduction in the number of nonhub-hub connections. When $r=2$ and $\alpha=1$, the routing cost increases by $4.46 \%$ with respect to the multiple allocation solution but is $13.17 \%$ cheaper than the single allocation solution. Hence, allowing each node to be allocated to two hubs rather than a single one brings a significant reduction in routing costs. Finally, we minimize the number of nonhub-hub connections (including the connections from a hub to itself) subject to an upper bound on the total transportation cost. For $\alpha=1$, the minimum number of nonhub-hub connections turn out to be 59 when we allow an increase of $1 \%$ in the transportation cost and 42 when we allow an increase of $5 \%$ over the cost of the multiple allocation solution. For $\alpha=0.8$, the number of nonhub-hub connections can be reduced to 46 and 34 by allowing $1 \%$ and $5 \%$ increases in the transportation cost, respectively. In Fig. 1, we can see the change in the number of nonhub-hub connections for different percentage increases allowed in the optimal multiple allocation routing cost.

As a result of this analysis, we conclude that allocation in hub networks is an important issue and the trade-off between fixed costs related to nonhub-hub connections and total transportation costs is interesting to analyze in designing hub networks. In this study, we present different problems and their associated models to be used in such an analysis.

To conclude this section, we briefly review the literature on multiple and single allocation $p$-hub median problems. For more

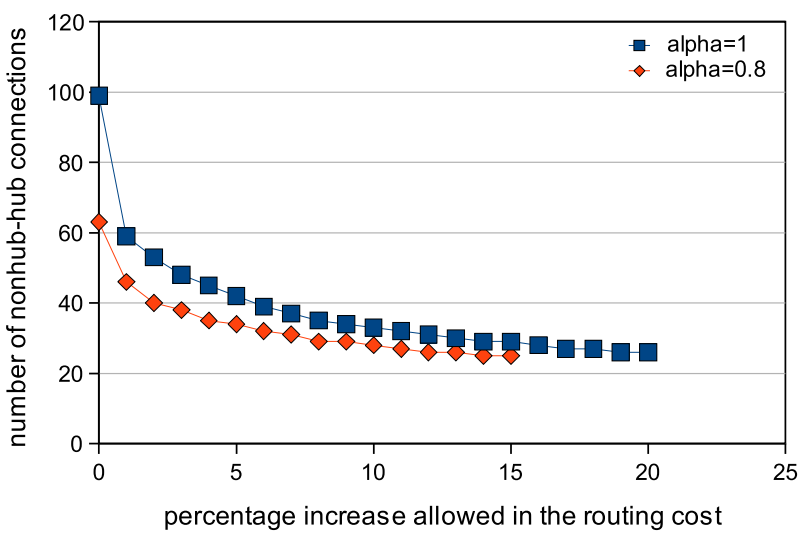

Fig. 1. The change in the number of nonhub-hub connections as the upper bound on the routing cost increases. 
details, we refer readers to two recent surveys: Campbell et al. (2002), and Alumur and Kara (2008).

Research on the single allocation $p$-hub median problem starts with the works of O'Kelly (1987). Different formulations are proposed by Campbell (1994), Ebery (2001), Ernst and Krishnamoorthy (1996), O'Kelly et al. (1996), Skorin-Kapov et al. (1996), and Sohn and Park (1997, 1998). Abdinnour-Helm (2001), Campbell (1996), Ernst and Krishnamoorthy (1996), Klincewicz (1991, 1992), O'Kelly (1987), Pirkul and Schilling (1998), Skorin-Kapov and Skorin-Kapov (1994), and Smith et al. (1996) present heuristic algorithms. O'Kelly et al. (1995) present a lower bounding procedure and Ernst and Krishnamoorthy (1998a) propose a branchand-bound method, where shortest-path problems are solved to compute lower bounds. Kratica et al. (2007) develop two genetic algorithms and Ilić et al. (2010) develop a variable neighborhood search. Sung and Jin (2001), and Wagner (2007) study the cluster hub location problem.

The first formulation for the multiple allocation $p$-hub median problem is given in Campbell (1992). Campbell (1994), Ernst and Krishnamoorthy (1998b), and Skorin-Kapov et al. (1996) propose alternative formulations. Campbell (1996) presents a greedy-interchange heuristic. Ernst and Krishnamoorthy (1998b) propose a heuristic algorithm based on shortest paths and a cut-and-branch algorithm. The same authors also propose a branch-and-bound algorithm using shortest paths (Ernst and Krishnamoorthy, 1998a). Preprocessing tools and ways to strengthen constraints are given in Boland et al. (2004).

A close relative of the $p$-hub median problem where the number of hubs to be opened is also a part of the decision process is called the hub location problem with fixed costs. The single allocation version of this problem is proposed by O'Kelly (1992) and later studied by Abdinnour-Helm (1998), Abdinnour-Helm and Venkataramanan (1998), Campbell (1994), Chen (2007), Cunha and Silva (2007), Labbé and Yaman (2004), Silva and Cunha (2009), and Topcuoglu et al. (2005). The multiple allocation version is studied by Boland et al. (2004), Camargo et al. (2008), Campbell (1994), Cánovas et al. (2007), Hamacher et al. (2004), Klincewicz (1996), Mayer and Wagner (2002), Marín (2005), and Marín et al. (2006).

The rest of the paper is organized as follows: In Section 2, we present two formulations for the UrApHMP and investigate the relationship of these formulations with two well-known formulations for the $p$-hub median problem. We discuss some problem variations and propose ways to model these in Section 3. In Section 4 , we report the results of our computational study and derive some insights on the effect of the allocation strategies on routing costs and locations of hubs in optimal solutions. We conclude the paper in Section 5.

\section{Mixed-integer programming formulations}

For the single and multiple allocation $p$-hub median problems, there are two types of formulations that are commonly used. The first of these formulations uses up to four index variables and is the tighter formulation. There is a flow variable for each pair of nodes $i$ and $j$ and each pair of possible hubs $k$ and $l$; this variable gives the proportion of the flow from node $i$ to node $j$ that travels from the origin node $i$ to hub $k$, and then to hub $l$, and finally to the destination node $j$. In the second type of formulations, the flow variables are aggregated by origin and/or destination, resulting in variables with at most three indices. These formulations are not as strong but have the advantage of fewer variables. There are other formulations that use fewer variables, however such formulations have weaker relaxation bounds and need to be strengthened with cutting planes to be efficient.

In this section, we provide two mixed-integer programming formulations for UrApHMP that extend the first two types of formula- tions mentioned above. We first present the notation. Let $N$ denote the set of demand points. For $i \in N$ and $j \in N, t_{i j}$ is the amount of traffic to be routed from node $i$ to node $j$ and $d_{i j}$ is the unit routing cost from node $i$ to node $j$. We assume that the routing costs satisfy the triangle inequality.

We use the following decision variables in our first model for UrApHMP. The variable $z_{i k}$ is 1 if node $i \in N$ is allocated to node $k \in N$ and 0 otherwise. If $z_{k k}$ is 1 , then node $k$ is allocated to itself, which means that a hub is opened at this node. The variable $f_{i j k l}$ is the proportion of the traffic from node $i \in N$ to node $j \in N$ that travels on the path $i \rightarrow k \rightarrow l \rightarrow j$ where $k$ and $l$ are hub nodes. Using these variables, the UrApHMP can be modeled as follows:

$$
\begin{array}{ll}
\min & \sum_{i \in N} \sum_{j \in N} \sum_{k \in N} \sum_{l \in N} t_{i j}\left(d_{i k}+\alpha d_{k l}+d_{l j}\right) f_{i j k l}, \\
\text { s.t. } & \sum_{k \in N} z_{i k} \leqslant r \quad \forall i \in N, \\
& z_{i k} \leqslant z_{k k} \quad \forall i, k \in N, \\
& \sum_{k \in N} z_{k k}=p, \\
& \sum_{k \in N} \sum_{l \in N} f_{i j k l}=1 \quad \forall i, j \in N \\
& \sum_{l \in N} f_{i j k l} \leqslant z_{i k} \quad \forall i, j, k \in N, \\
& \sum_{k \in N} f_{i j k l} \leqslant z_{j l} \quad \forall i, j, l \in N, \\
& f_{i j k l} \geqslant 0 \quad \forall i, j, k, l \in N, \\
& z_{i k} \in\{0,1\} \quad \forall i, k \in N .
\end{array}
$$

Constraints (2) ensure that a node is allocated to at most $r$ hubs and constraints (3) ensure that nodes can only be allocated to hubs. Due to constraint (4), $p$ nodes are selected as hubs. Constraints (5) impose the requirement that the traffic between any origin destination pair is routed entirely. Finally, constraints (6) and (7) ensure that if the flow of origin-destination pair $i, j$ travels from hub $k$ to hub $l$, then $i$ is allocated to hub $k$ and $j$ is allocated to hub $l$. Constraints (8) and (9) are nonnegativity and integrality constraints. Note here that there exists an optimal solution where the vector $f$ has entries 0 and 1 , since each traffic demand is routed on a minimum-cost path in the resulting network.

Let the above model be UrApHMP1. It has $O\left(|N|^{4}\right)$ variables and $O\left(|N|^{3}\right)$ constraints. Next, we discuss its relationship to the strong 4 -index formulations for the single and multiple allocation $p$-hub median problems. First, notice that there exists an optimal solution to this model, with $\sum_{k \in N} z_{i k}=r$ for all $i \in N$. If $r=1$, then we have $\sum_{k \in N} z_{i k}=1$ for all $i \in N$. Now, constraints (5) imply that constraints (6) and (7) have to be tight, i.e., $\sum_{l \in N} f_{i j k l}=z_{i k}$ for all $i, j, k \in N$ and $\sum_{k \in N} f_{i j k l}=z_{j l}$ for all $i, j, l \in N$. Hence, when $r=1$, UrApHMP1 is equivalent to the strong formulation for the single allocation $p$-hub median problem given by Skorin-Kapov et al. (1996). This formulation is as follows:

$$
\begin{array}{ll}
\min & \sum_{i \in N} \sum_{j \in N} \sum_{k \in N} \sum_{l \in N} t_{i j}\left(d_{i k}+\alpha d_{k l}+d_{l j}\right) f_{i j k l}, \\
\text { s.t. } & (3),(4),(8), \text { and }(9), \\
& \sum_{k \in N} z_{i k}=1 \quad \forall i \in N, \\
& \sum_{l \in N} f_{i j k l}=z_{i k} \quad \forall i, j, k \in N \\
& \sum_{k \in N} f_{i j k l}=z_{j l} \quad \forall i, j, l \in N .
\end{array}
$$

If $r=p$, then there exists an optimal solution to UrApHMP1 and to its linear programming relaxation with $z_{i k}=z_{k k}$ for all $i, k \in N$. Then we 
can remove the variables $z_{i k}$ 's for $i, k \in N$ with $i \neq k$. Hence, constraints (6) and (7) are the same as $\sum_{l \in N} f_{i j k l} \leqslant z_{k k}$ for all $i, j, k \in N$ and $\sum_{k \in N} f_{i j k l} \leqslant z_{l l}$ for all $i, j, l \in N$. The resulting formulation is the same as the strong formulation for the multiple allocation $p$-hub median problem given by Skorin-Kapov et al. (1996):

$$
\begin{array}{ll}
\min & \sum_{i \in N} \sum_{j \in N} \sum_{k \in N} \sum_{l \in N} t_{i j}\left(d_{i k}+\alpha d_{k l}+d_{l j}\right) f_{i j k l}, \\
\text { s.t. } & (4),(5), \text { and }(8), \\
& \sum_{l \in N} f_{i j k l} \leqslant z_{k k} \quad \forall i, j, k \in N, \\
& \sum_{k \in N} f_{i j k l} \leqslant z_{l l} \quad \forall i, j, l \in N, \\
& z_{k k} \in\{0,1\} \quad \forall k \in N .
\end{array}
$$

This formulation is later strengthened by replacing constraints (15) and (16) with (see Hamacher et al., 2004) $\sum_{l \in N} f_{i j k l}+\sum_{l \in N \backslash\{k\}} f_{i j l k} \leqslant z_{k k}$ for all $i, j, k \in N$. These inequalities are valid for the UrApHMP but it is not possible to obtain a valid formulation by replacing constraints (6) and (7) with these inequalities for $r \leqslant p-1$.

Next, we extend the aggregated flow formulation of Ernst and Krishnamoorthy (1998b) for the multiple allocation $p$-hub median problem to UrApHMP. We define the following variables. Variable $w_{k}^{i}$ is the flow from node $i \in N$ to hub $k \in N, y_{k l}^{i}$ is the flow that originates at node $i \in N$ and that travels from hub $k \in N$ to hub $l \in N \backslash\{k\}$, and $x_{l j}^{i}$ is the flow that originates at node $i \in N$ and that travels from hub $l \in N$ to destination node $j \in N$. Then UrApHMP can be formulated as follows:

$$
\begin{array}{ll}
\min & \sum_{i \in N}\left(\sum_{k \in N} d_{i k} w_{k}^{i}+\sum_{k \in N} \sum_{l \in N \backslash\{k\}} \alpha d_{k l} y_{k l}^{i}+\sum_{j \in N} \sum_{l \in N} d_{l j} x_{l j}^{i}\right), \\
\text { s.t. } & (2)-(4) \text { and }(9), \\
& \sum_{k \in N} w_{k}^{i}=\sum_{j \in N} t_{i j} \quad \forall i \in N, \\
& \sum_{l \in N \backslash\{k\}} y_{k l}^{i}-\sum_{l \in N \backslash\{k\}} y_{l k}^{i}=w_{k}^{i}-\sum_{j \in N} x_{k j}^{i} \quad \forall i, k \in N, \\
& \sum_{l \in N} x_{l j}^{i}=t_{i j} \quad \forall i, j \in N, \\
& w_{k}^{i} \leqslant \sum_{j \in N} t_{i j} z_{i k} \quad \forall i, k \in N, \\
& x_{l j}^{i} \leqslant t_{i j} z_{j l} \quad \forall i, j, l \in N, \\
& w_{k}^{i} \geqslant 0 \quad \forall i, k \in N, \\
& x_{k j}^{i} \geqslant 0 \quad \forall i, j, k \in N, \\
& y_{k l}^{i} \geqslant 0 \quad \forall i, k, l \in N .
\end{array}
$$

Here, constraints (19)-(21) are flow balance constraints. Constraints (19) ensure that for each node $i$, the total traffic originating at node $i$ travels from $i$ to hubs. Due to constraints (20), the net flow that originates at node $i$ and that travels out of hub node $k$ towards other hubs is equal to the flow of node $i$ that travels from $i$ to $k$ minus the flow of node $i$ that travels from hub $k$ towards the destination nodes. Finally, constraints (21) ensure that the flow that originates at node $i$ and that travels from hub nodes to destination node $j$ is equal to the traffic demand from node $i$ to node $j$. By constraints (22), the flow from origin node $i$ to hub node $k$ is forced to be zero if node $i$ is not allocated to hub $k$. Finally, constraints (23) ensure that the flow of origin node $i$ that travels from hub $l$ to destination node $j$ cannot be positive unless node $j$ is not allocated to hub $l$. Constraints (24)-(26) are nonnegativity constraints for flow variables.

Let this model be UrApHMP2. Here we have $O\left(|N|^{3}\right)$ variables and $O\left(|N|^{3}\right)$ constraints. As in the case of UrApHMP1, UrApHMP2 is equivalent to Ernst and Krishnamoorthy's formulation for $r=1$ and $r=p$. If $r=1$, we have $\sum_{k \in N} z_{i k}=1$ for all $i \in N$. Then, constraints (19) and (22) imply that $w_{k}^{i}=\sum_{j \in N} t_{i j} z_{i k}$ for all $i \in N$ and $k \in N$. Similarly, constraints (21) and (23) imply that $x_{l j}^{i}=t_{i j} z_{j l}$ for all $i, j, l \in N$. Hence, we can substitute for variables $w_{k}^{i}$ and $x_{l j}^{i}$ and obtain the formulation

$$
\begin{aligned}
& \min \sum_{i \in N}\left(\sum_{k \in N} d_{i k} \sum_{j \in N} t_{i j} z_{i k}+\sum_{k \in N} \sum_{l \in N \backslash\{k\}} \alpha d_{k l} y_{k l}^{i}+\sum_{j \in N} \sum_{l \in N} d_{l j} t_{i j} z_{j l}\right), \\
& \text { s.t. (3), (4), (9), (11), (26), } \\
& \sum_{l \in N \backslash\{k\}} y_{k l}^{i}-\sum_{l \in N \backslash\{k\}} y_{l k}^{i}=\sum_{j \in N} t_{i j} z_{i k}-\sum_{j \in N} t_{i j} z_{j k} \quad \forall i, k \in N,
\end{aligned}
$$

which is the same as the formulation given in Ernst and Krishnamoorthy (1996).

For $r=p$, again as $z_{i k}=z_{k k}$ for all $i, k \in N$, we can remove these variables and obtain the following formulation:

$$
\begin{array}{ll}
\min & \sum_{i \in N}\left(\sum_{k \in N} d_{i k} w_{k}^{i}+\sum_{k \in N} \sum_{l \in N \backslash\{k\}} \alpha d_{k l} y_{k l}^{i}+\sum_{j \in N} \sum_{l \in N} d_{l j} x_{l j}^{i}\right), \\
\text { s.t } \quad(4), \quad(17),(19)-(21),(24)-(26), \\
& w_{k}^{i} \leqslant \sum_{j \in N} t_{i j} z_{k k} \quad \forall i, k \in N, \\
& x_{l j}^{i} \leqslant t_{i j} z_{l l} \quad \forall i, j, l \in N .
\end{array}
$$

This formulation is the same as that given in Ernst and Krishnamoorthy (1998b).

Hence, for the special cases of $r=1$ and $r=p$, the formulations presented in this section, UrApHMP1 and UrApHMP2, have the same linear programming relaxation bounds as the formulations of Skorin-Kapov et al. (1996), and Ernst and Krishnamoorthy (1996, 1998b), respectively.

\section{Modeling problem variations}

In this section, we discuss ways to model several problem variations.

In most practical applications, nodes' incoming and outgoing traffic demands may have large variations. In such cases, network planners may be interested in networks where nodes with high demand values are allocated to more hubs and nodes with low demand values are allocated to fewer hubs. For each node $i \in N$, we have a value $r_{i}$, which is an upper bound on the number of hubs that node $i$ can be allocated to. This variation can be easily modeled by replacing constraints (2) with $\sum_{k \in N} z_{i k} \leqslant r_{i}$ for all $i \in N$.

As we have seen with the CAB data in the Introduction, it may be interesting to find solutions with minimum number of nonhub-hub connections subject to a constraint that imposes an upper bound on the transportation cost. The resulting problem may be solved with different values of upper bounds to make sensitivity analysis and see the trade-off between fixed costs and transportation costs. Let $c_{i k}$ be the fixed cost for establishing a nonhub-hub connection between node $i \in N$ and hub $k \in N$ and $U$ be an upper bound on the transportation cost. The problem of minimizing the fixed costs associated with the nonhub-hub connections such that the transportation cost does not exceed $U$ can be modeled as follows:

$$
\begin{array}{ll}
\min & \sum_{i \in N} \sum_{k \in N} c_{i k} z_{i k}, \\
\text { s.t. } & (3)-(9), \\
& \sum_{i \in N} \sum_{j \in N} \sum_{k \in N} \sum_{l \in N} t_{i j}\left(d_{i k}+\alpha d_{k l}+d_{l j}\right) f_{i j k l} \leqslant U .
\end{array}
$$

An alternative model can be obtained as follows: 
$\min \sum_{i \in N} \sum_{k \in N} c_{i k} z_{i k}$

s.t. (3), (4), (9), (19)-(26),

$$
\sum_{i \in N}\left(\sum_{k \in N} d_{i k} w_{k}^{i}+\sum_{k \in N} \sum_{l \in N \backslash\{k\}} \alpha d_{k l} y_{k l}^{i}+\sum_{j \in N} \sum_{l \in N} d_{l j} x_{l j}^{i}\right) \leqslant U .
$$

In addition, let $T_{i k}$ be the minimum flow that should be routed on the nonhub-hub connection between node $i \in N$ and hub $k \in N$ for such a connection to exist. Then the problem of minimizing the sum of the fixed costs of nonhub-hub connections and the routing cost subject to the constraint that the flow on nonhub-hub connections should be at least as large as the threshold values can be modeled as

$$
\begin{array}{ll}
\min & \sum_{i \in N} \sum_{k \in N} c_{i k} z_{i k}+\sum_{i \in N} \sum_{j \in N} \sum_{k \in N} \sum_{l \in N} t_{i j}\left(d_{i k}+\alpha d_{k l}+d_{l j}\right) f_{i j k l}, \\
\text { s.t. } & (3)-(9), \\
& \sum_{j \in N} \sum_{l \in N}\left(t_{i j} f_{i j k l}+t_{j i} f_{j i l k}\right) \geqslant T_{i k} z_{i k} \quad \forall i, k \in N .
\end{array}
$$

Campbell (1994) models the flow threshold requirements as

$\sum_{j \in N} \sum_{l \in N}\left(t_{i j} f_{i j k l}+t_{j i} f_{j i l k}\right) \geqslant T_{i k}-M\left(1-z_{i k}\right) \quad \forall i, k \in N$,

where $M$ is a large number. Constraints (32) dominate constraints (33).

Our second model can be modified for this problem as follows:

$$
\begin{aligned}
& \min \sum_{i \in N} \sum_{k \in N} c_{i k} z_{i k}+\sum_{i \in N}\left(\sum_{k \in N} d_{i k} w_{k}^{i}+\sum_{k \in N} \sum_{l \in N \backslash\{k\}} \alpha d_{k l} y_{k l}^{i}+\sum_{j \in N} \sum_{l \in N} d_{l j} x_{l j}^{i}\right), \\
& \text { s.t. }(3),(4),(9),(19)-(26), \\
& \quad w_{k}^{i}+\sum_{j \in N} x_{k i}^{j} \geqslant T_{i k} z_{i k} \quad \forall i, k \in N .
\end{aligned}
$$

We note here that with the flow threshold constraints, it is not necessarily true that there exists an optimal solution where the traffic demand between a pair of nodes follows the same path, i.e., $f_{i j k l}$ 's are zero and one. If such a nonsplitting is required, then integrality should be imposed explicitly for these variables. In this case, we need extra variables in the second model because it uses aggregate flow variables.

If service quality is one of the issues in the network design process, then the decision maker may be interested in optimizing the worst service quality in the network or ensuring a minimum service quality by imposing restrictions on delivery times, transportation costs, and/or path lengths. The well-known $p$-hub center problem is an example of the first kind, where the aim is to minimize the largest transportation cost or distance among all pairs of nodes (see, e.g., Campbell, 1994; Ernst et al., 2009; Kara and Tansel, 2000; O'Kelly and Miller, 1991). Another example of hub location problems where one is interested in the worst-case performance is the latest-arrival hub location problem, introduced by Kara and Tansel (2001). The authors consider delivery times as a measure of service quality and aim at minimizing the longest delivery time in a single allocation hub network. They remark that if a single vehicle is used on each link, then the vehicles departing from a hub node towards other hubs should wait for the arrival of vehicles from nodes that are allocated to this hub. Similarly, the vehicles departing from a hub node towards the destination nodes that are allocated to this hub should wait for the arrival of vehicles from other hubs and from the nodes that are allocated to this hub. Hence, the delivery time is composed of travel times and waiting times. Later, Wagner (2004) show that this problem is equivalent to the $p$-hub center problem, as the vehicles that travel on the path that has the longest delivery time do not wait.

In our study, we are interested in minimizing the total routing cost under some service quality constraints as done in Campbell (2009), Yaman et al. (2007), and Yaman (2009).

If restrictions are imposed on path lengths or transportation costs (see Campbell, 2009), they can be handled easily with the UrApHMP1. For each origin-destination pair $i, j$, we define a set of eligible hub pairs $k, l$, through which the traffic from $i$ to $j$ can be routed. Suppose that upper bounds are to be imposed on path lengths. Let $\tau_{k l}$ be the length of connection from node $k$ to node $l$ and $\beta$ be an upper bound on the path length. Then we define $P_{i j}=\left\{(k, l): k, l \in N, \tau_{i k}+\tau_{k l}+\tau_{l j} \leqslant \beta\right\}$ for each pair of nodes $i$ and $j$ in $N$. Now path-length constraints may be imposed by adding

$f_{i j k l}=0 \quad \forall i, j, k, l \in N:(k, l) \notin P_{i j}$,

to UrApHMP1, and as such, paths that do not satisfy the path-length constraints are avoided. The same approach can be used to impose upper bounds on transportation costs. We note here that imposing similar constraints in UrApHMP2 requires defining additional variables because flow variables are aggregate variables and because the fact that $z_{i k}=1$ and $z_{j l}=1$ does not imply that the flow from node $i$ to node $j$ travels through hubs $k$ and $l$ unless $r=1$.

To ensure a given service quality, delivery time restrictions may also be imposed (see Yaman et al., 2007; Yaman, 2009). In this case, let $\tau_{k l}$ be the time to travel from $k$ to $l, \bar{\alpha}$ be a discount factor for hubto-hub connections, and $\beta$ be an upper bound on the delivery time. We assume that the travel times satisfy triangle inequality. As done above, we can define a set of eligible hub pairs, however, this approach may not sufficient. Consider the following case: Suppose that nodes 1 and 2 are hubs and the traffic from node 3 to node 4 and from node 5 to node 6 are routed through these hubs. The travel times are $\tau_{31}=40, \tau_{51}=10, \tau_{12}=50, \tau_{24}=10$, and $\tau_{26}=40$. We take $\bar{\alpha}=1$ and $\beta=100$. Then the path from node 3 to node 4 and the path from node 5 to node 6 have lengths 100 . However, as the vehicle at hub 1 has to wait for the traffic from nodes 3 and 5 to arrive before it can depart towards hub 2, it departs at time 40 , arrives at node 2 at time 90 , and at nodes 4 and 6 at times 100 and 130 , respectively. Such a routing is feasible when restrictions are imposed on path lengths but not on delivery times. As was remarked by Wagner (2004), such a situation does not arise for the single allocation problem, where traffic demands exist between all pairs of nodes, since in that case, the traffic from node 3 to node 6 also needs to travel through hubs 1 and 2 and the length of the resulting path is 130 , which in turn implies that such an allocation is infeasible. Hence, for single allocation, imposing restrictions on path lengths, transportation costs, and delivery times are equivalent, whereas for $r \geqslant 2$, these may not be equivalent.

As we explained above, in a single allocation problem where there is a traffic demand between any pair of nodes and a single vehicle on each link, the vehicles that depart from a hub $k$ towards other hubs need to wait for the arrival of vehicles from nodes that are allocated to $k$. Hence, the earliest departure time is the same for all vehicles that depart from hub $k$ towards other hubs. However, for $r \geqslant 2$, this is not true anymore. To model this, we define variable $D_{k l}$ to be the earliest time that the vehicle travelling on the link from hub $k$ to hub $l$ can depart. This time cannot be earlier than the arrival of vehicles from nodes that are allocated to hub $k$ and that have traffic demands that travel through hubs $k$ and $l$. This requirement can be expressed with constraints

$D_{k l} \geqslant \tau_{i k} f_{i j k l} \quad \forall i, j, k, l \in N$.

The vehicle traveling on the link from hub $k$ to hub $l$ arrives at hub $l$ at the earliest at time $D_{k l}+\bar{\alpha} \tau_{k l}$. If node $j$ is allocated to hub $l$, then the vehicle traveling from $l$ to $j$ needs to wait for the arrival of the vehicle from a hub $k$ in the event that there is traffic demand that 
has its destination at $j$ and that travels through hubs $k$ and $l$. Hence, the vehicle that departs from $l$ towards $j$ can arrive at $j$ at the earliest at time $\max _{k \in N}\left(\left(D_{k l}+\bar{\alpha} \tau_{k l}+\tau_{l j}\right) \max _{i \in N} f_{i j k l}\right)$. We would like this arrival time to be no later than $\beta$, hence we use constraints

$\left(D_{k l}+\bar{\alpha} \tau_{k l}+\tau_{l j}\right) f_{i j k l} \leqslant \beta \quad \forall i, j, k, l \in N$.

Finally, we explicitly require $f_{i j k l}$ 's to be integers with

$f_{i j k l} \in\{0,1\} \quad \forall i, j, k, l \in N$.

Constraints (35) can be linearized as

$D_{k l}+\bar{\alpha} \tau_{k l}+\tau_{l j} f_{i j k l} \leqslant \beta \quad \forall i, j, k, l \in N$

Let $i, j, k, l \in N$. If $f_{i j k l}=1$, then the left-hand sides of (35) and (37) are equal. If $f_{i j k l}=0$, then the left hand side of (35) is equal to zero whereas the left-hand side of (37) is equal to $D_{k l}+\bar{\alpha} \tau_{k l}$. If there exist $i^{\prime}$ and $j^{\prime}$ such that $i^{\prime} \neq i$ or $j^{\prime} \neq j$ with $f_{i^{\prime} j^{\prime} k l}=1$, then constraint (37) for $i^{\prime}, j^{\prime}, k$, and $l$ states that $D_{k l}+\bar{\alpha} \tau_{k l}+\tau_{l^{\prime}} \leqslant \beta$, making constraint (37) for $i, j, k$, and $l$ redundant. On the other hand, if $f_{i^{\prime} j^{\prime} k l}=0$ for all $i^{\prime}$ and $j^{\prime}$, then there exists an optimal solution such that $D_{k l}=0$ and (37) for $i, j, k$ and $l$ reduces to $\bar{\alpha} \tau_{k l} \leqslant \beta$. As $\bar{\alpha} \tau_{k l}$ is a lower bound on the travel time from node $k$ to node $l$, this needs to be satisfied for the feasibility of the problem.

In summary, upper bounds on delivery times can be incorporated into UrApHMP1 by adding constraints (34) and (37) and by replacing (8) with (36). The resulting formulation is valid for any integer $1 \leqslant r \leqslant p$.

Aykin (1994, 1995), Sung and Jin (2001), and Wagner (2007) consider the possibility of non-stop service between origin-destination pairs. This can be incorporated into our model UrApHMP1 in the following way. Let $\hat{d}_{i j}$ denote the unit routing cost from node $i \in N$ to node $j \in N$ for non-stop service. We define $\hat{f}_{i j}$ to be the proportion of the demand from origin $i \in N$ to destination $j \in N$ routed on a non-stop service. We replace the objective function (1) with

$\min \sum_{i \in N} \sum_{j \in N} \sum_{k \in N} \sum_{l \in N} t_{i j}\left(d_{i k}+\alpha d_{k l}+d_{l j}\right) f_{i j k l}+\sum_{i \in N} \sum_{j \in N} t_{i j} \hat{d}_{i j} \hat{f}_{i j}$.

In addition, we replace constraints (5) with

$\sum_{k \in N} \sum_{l \in N} f_{i j k l}+\hat{f}_{i j}=1 \quad \forall i, j \in N$

and add constraints

$\hat{f}_{i j} \geqslant 0 \quad \forall i, j \in N$.

As in the case of UrApHMP1, there exists an optimal solution where $f$ and $\hat{f}$ are integral.

In some applications, the demand from an origin to a destination may depend on the number of hubs traversed. Airlines is a good example to this case; most clients prefer non-stop flights. In such an application, minimizing the total routing cost is not a reasonable objective as it would have a tendency to minimize the demand captured. In this case, the decision maker may be interested in maximizing profit. Let $p_{i j k l}$ be the profit generated for the origin-destination pair $(i, j)$ if the path from origin $i \in N$ to destination $j \in N$ goes through hubs $k \in N$ and $l \in N$. Similarly, let $\hat{p}_{i j}$ be the profit generated if there is a non-stop service from origin $i$ to destination $j$. Now, this variant can be modeled as follows:

$$
\begin{aligned}
& \max \sum_{i \in N} \sum_{j \in N} \sum_{k \in N} \sum_{l \in N} p_{i j k l} f_{i j k l}+\sum_{i \in N} \sum_{j \in N} \hat{p}_{i j} \hat{f}_{i j}, \\
& \text { s.t. }(2)-(4),(6)-(9),(38) \text { and (39). }
\end{aligned}
$$

Note again that, even though we do not impose integrality on vectors $f$ and $\hat{f}$, there exists an optimal solution where each origin-destination pair is routed on a single path.

\section{Computational results}

In this section, we report the outcomes of three computational experiments. In the first experiment, we continue our analysis on routing costs that started in the Introduction. We investigate how the routing cost changes as we change the allocation strategy. In the second experiment, we are interested in the location of hubs in optimal solutions for different allocation strategies. Finally, in the third experiment, we compare our formulations in terms of the strength of their linear relaxation bounds and solution times.

In these experiments, we solve instances generated using $C A B$, Australia Post (AP), and Turkey data. We use the CAB data with 25 nodes, $p=3,4,5$, and $\alpha=1,0.8,0.6,0.4,0.2$. The AP data is introduced by Ernst and Krishnamoorthy (1996) and consists of coordinate and flow data for 200 nodes that correspond to postcode districts. Here, the collection cost is computed by multiplying the distance by 3 , the distribution cost is computed by multiplying the distance by 2 , and the hub-to-hub routing cost is discounted with $\alpha=0.75$. The flow matrix is not symmetric and flows from a node to itself are positive. Smaller-sized problems can be generated using a code made available by the authors. We generated instances with $n=50,75$ and varied $p$ to be 3,4 , and 5. In the Turkey data, we have 81 nodes, each corresponding to a city. The unit routing costs are taken to be equal to distances between cities. The traffic demand between a pair of cities is computed by multiplying the populations of the respective two cities, dividing by a constant, and rounding down. Twenty-one cities are candidates to be hubs. This is the same dataset as the one used by Yaman (2009). We take $\alpha=0.9$ and $p$ to be in $\{5, \ldots, 10\}$.

\subsection{The effect of allocation strategies on total routing costs}

In this section, we continue analyzing the effect of allocation strategies on the total routing costs. Let $\operatorname{cost}_{r}$ be the minimum routing cost for a given $r$ value. We compute how much more expensive the $r$-allocation solution is compared to the multiple allocation solution by $\exp _{r}=100 \frac{\operatorname{cost}_{r}-\operatorname{cost}_{p}}{\operatorname{cost}_{p}}$ and how much cheaper the $r$-allocation solution is compared to the single allocation solution by cheap $_{r}=100 \frac{\text { cost }_{1}-\text { cost }_{r}}{\text { cost }_{1}}$.

In Table 1, we report exp $\exp _{r}$ and cheap for $r=3$ and $r=2$ and $\exp _{1}$ for the CAB data for different values of $p$ and $\alpha$. We first observe here that for $\alpha$ fairly large, single allocation solutions are considerably more costly than multiple allocation solutions. Second, we observe that it is possible to obtain a significant cost reduction by allowing nodes to be allocated to one or two more hubs. For

Table 1

Comparison of total routing costs for $r=1,2,3, p$ for the CAB data with $n=25$.

\begin{tabular}{rllrlrrr}
\hline$p$ & $\alpha$ & $r=3$ & & & $r=2$ & $r=1$ \\
& & exp $_{3}$ & cheap $_{3}$ & & exp $_{2}$ & cheap $_{2}$ & exp $_{1}$ \\
\hline 3 & 1 & 0 & 15.48 & & 1.06 & 14.58 & 18.31 \\
3 & 0.8 & 0 & 11.98 & & 0.34 & 11.68 & 13.61 \\
3 & 0.6 & 0 & 8.16 & 0.08 & 8.09 & 8.88 \\
3 & 0.4 & 0 & 4.66 & 0 & 4.66 & 4.89 \\
3 & 0.2 & 0 & 1.88 & 0 & 1.88 & 1.92 \\
4 & 1 & 0.34 & 16.60 & 2.95 & 14.44 & 20.32 \\
4 & 0.8 & 0 & 12.49 & 1.75 & 10.97 & 14.28 \\
4 & 0.6 & 0 & 7.75 & 1.30 & 6.55 & 8.40 \\
4 & 0.4 & 0 & 4.19 & 0.61 & 3.60 & 4.38 \\
4 & 0.2 & 0 & 1.77 & 0 & 1.77 & 1.80 \\
5 & 1 & 0.65 & 16.34 & 4.46 & 13.17 & 20.30 \\
5 & 0.8 & 0.21 & 11.78 & 2.70 & 9.59 & 13.59 \\
5 & 0.6 & 0.34 & 7.88 & 1.72 & 6.62 & 8.93 \\
5 & 0.4 & 0.25 & 4.19 & 0.81 & 3.66 & 4.64 \\
5 & 0.2 & 0 & 1.56 & 0.06 & 1.49 & 1.58 \\
\hline
\end{tabular}


Table 2

Comparison of total routing costs for $r=1,2,3, p$ for the AP data.

\begin{tabular}{|c|c|c|c|c|c|c|c|}
\hline \multirow[t]{2}{*}{$n$} & \multirow[t]{2}{*}{$p$} & \multirow{2}{*}{$\begin{array}{l}r=p \\
\text { cheap }_{p}\end{array}$} & \multicolumn{2}{|l|}{$r=3$} & \multicolumn{2}{|l|}{$r=2$} & \multirow{2}{*}{$\begin{array}{l}r=1 \\
\exp _{1}\end{array}$} \\
\hline & & & $\exp _{3}$ & cheap $_{3}$ & $\exp _{2}$ & cheap $_{2}$ & \\
\hline \multirow[t]{3}{*}{50} & 3 & 1.61 & 0 & 1.61 & 0.09 & 1.52 & 1.64 \\
\hline & 4 & 1.55 & 0.07 & 1.48 & 0.18 & 1.38 & 1.58 \\
\hline & 5 & 2.23 & 0.08 & 2.16 & 0.28 & 1.96 & 2.28 \\
\hline \multirow[t]{3}{*}{75} & 3 & 1.91 & 0 & 1.91 & 0.12 & 1.79 & 1.95 \\
\hline & 4 & 2.17 & 0.06 & 2.10 & 0.20 & 1.98 & 2.22 \\
\hline & 5 & 2.68 & 0.02 & 2.67 & 0.35 & 2.35 & 2.76 \\
\hline
\end{tabular}

instance, for $p=4$ and $\alpha \leqslant 0.8$, the optimal solutions with $r=3$ have the same cost as the multiple allocation solutions.

In Table 2, we report the percentage differences between total routing costs with different allocation strategies, i.e., $r=1,2,3, p$, for the AP data with 50 and 75 nodes and $p=3,4,5$ hubs. Here we observe that the single allocation solutions are around 1-3\% more expensive than the multiple allocation solutions. The largest percentage differences, $2.28 \%$ for $n=50$ and $2.76 \%$ for $n=75$, occur for $p=5$. For $p=5$, when we allow each node to be allocated to two hubs rather than force the single allocation requirement, we obtain solutions that are only $0.28 \%$ and $0.35 \%$ more expensive than the multiple allocation solutions for $n=50$ and $n=75$, respectively. These solutions are $1.96 \%$ and $2.35 \%$ cheaper than the respective single allocation solutions. Further, when we allow a node to be allocated to three hubs, then the corresponding optimal solutions are only $0.08 \%$ and $0.02 \%$ more expensive than the multiple allocation solutions for $n=50$ and $n=75$, respectively.

For the Turkey data, we take $p=5, \ldots, 10$. Here we were not able to solve optimally the single and double allocation problems for most of our instances. Hence, we report the results for $r=3,4,5$. The solver stopped without proving optimality for $p=9$ and $r=3$, with a final gap of $0.06 \%$; we use the final upper bound reported by the solver for this instance. In Table 3 , for each value of $p$ and $r$, we report how much more expensive the $r$-allocation solution is compared to the multiple allocation solution. Here we see that even with 10 hubs, restricting the allocation of nodes to at most three hubs results in a $1.15 \%$ increase in the total routing cost. With $r=4$, the $r$-allocation solution is at most $0.29 \%$ more expensive than the multiple allocation solution. For $p=5,6,7$, restricting each node to be allocated to at most five nodes does not increase the total costs. For larger $p$ values, the increase is at most $0.08 \%$.

We can conclude that we have similar results for the three datasets that we used in this experiment. Allowing a node to be allocated to more than one hub results in significant improvements in routing costs. Most of this improvement can be achieved, however, even when the single-allocation requirement is relaxed, by allowing nodes to be allocated to a few hubs rather than all hubs.

\subsection{The effect of allocation strategies on hub locations in optimal solutions}

In this experiment, we investigate the effect of allocation strategies on hub locations. We use all three datasets in this

Table 3

Comparison of total routing costs for $r=3,4,5, p$ for the Turkey data.

\begin{tabular}{llll}
\hline$p$ & $\exp _{3}$ & $\exp _{4}$ & $\exp _{5}$ \\
\hline 5 & 0.48 & 0.01 & 0.00 \\
6 & 0.91 & 0.07 & 0.00 \\
7 & 1.12 & 0.19 & 0.00 \\
8 & 1.16 & 0.17 & 0.06 \\
9 & 1.37 & 0.29 & 0.08 \\
10 & 1.15 & 0.28 & 0.08 \\
\hline
\end{tabular}

experiment. The results are given in Tables 4-6 for the CAB, AP, and Turkey data, respectively.

For the CAB data with $p=3$, we observe that the value of $r$ affects the hub locations only slightly when $\alpha$ is small. For $\alpha=0.2$, the hub located at node 4 (Chicago) in the single allocation solution moves to node 21 (St.Louis) for $r=2$, 3. A similar situation occurs for $\alpha=0.4$ and 0.6 , where the hub located at node 18 (Philadelphia) moves to node 17 (New York) and the hub located at node 2 (Baltimore) moves to node 17 , respectively. For larger $\alpha$ values, the changes are more significant. For instance, with $\alpha=1$, hubs are located at nodes 4, 8 (Denver), and 20 (Pittsburgh) in the single allocation solution, whereas they are located at 12 (Los Angeles), 18 and 21 for $r=2,3$. We see that two hubs move away from the center as $r$ increases.

Table 4

Hub locations in optimal solutions for the CAB data with $n=25$.

\begin{tabular}{llllll}
\hline$p$ & $\alpha$ & $r=1$ & $r=2$ & $r=3$ & $r=p$ \\
\hline 3 & 1 & $4,8,20$ & $12,18,21$ & $12,18,21$ & $12,18,21$ \\
3 & 0.8 & $2,4,12$ & $12,18,21$ & $4,12,17$ & $4,12,17$ \\
3 & 0.6 & $2,4,12$ & $4,12,17$ & $4,12,17$ & $4,12,17$ \\
3 & 0.4 & $4,12,18$ & $4,12,17$ & $4,12,17$ & $4,12,17$ \\
3 & 0.2 & $4,12,17$ & $12,17,21$ & $12,17,21$ & $12,17,21$ \\
4 & 1 & $4,7,8,20$ & $4,12,13,18$ & $1,4,12,17$ & $1,4,12,17$ \\
4 & 0.8 & $1,4,12,18$ & $4,12,13,17$ & $1,4,12,17$ & $1,4,12,17$ \\
4 & 0.6 & $1,4,12,17$ & $1,4,12,17$ & $1,4,12,17$ & $1,4,12,17$ \\
4 & 0.4 & $1,4,12,17$ & $4,12,17,24$ & $4,12,17,24$ & $4,12,17,24$ \\
4 & 0.2 & $4,12,17,24$ & $4,12,17,24$ & $4,12,17,24$ & $4,12,17,24$ \\
5 & 1 & $1,2,4,7,8$ & $1,4,7,12,18$ & $1,4,7,12,17$ & $1,4,7,12,17$ \\
5 & 0.8 & $1,4,7,12,18$ & $1,4,7,12,17$ & $1,4,7,12,17$ & $4,7,12,17,24$ \\
5 & 0.6 & $4,7,12,14,17$ & $4,7,12,17,24$ & $4,7,12,17,24$ & $4,7,12,14,17$ \\
5 & 0.4 & $4,7,12,14,17$ & $4,7,12,14,17$ & $4,7,12,14,17$ & $4,7,12,14,17$ \\
5 & 0.2 & $4,17,12,14,17$ & $4,7,12,14,17$ & $4,7,12,14,17$ & $4,7,12,14,17$ \\
\hline
\end{tabular}

Table 5

Hub locations in optimal solutions for the AP data.

\begin{tabular}{llllll}
\hline$n$ & $p$ & $r=1$ & $r=2$ & $r=3$ & $r=p$ \\
\hline 50 & 3 & $14,28,35$ & $14,28,35$ & $14,28,35$ & $14,28,35$ \\
& 4 & $14,28,33,35$ & $14,28,32,35$ & $14,28,32,35$ & $14,28,32,35$ \\
& 5 & $4,14,28,33,35$ & $4,14,28,32,35$ & $4,14,28,32,35$ & $4,14,28,32,35$ \\
75 & 3 & $21,40,52$ & $21,41,52$ & $21,41,52$ & $21,41,52$ \\
& 4 & $21,40,49,52$ & $21,41,48,52$ & $21,41,48,52$ & $21,41,48,52$ \\
& 5 & $5,22,42,49,52$ & $5,22,42,48,52$ & $5,22,42,48,52$ & $5,22,42,48,52$ \\
\hline
\end{tabular}

Table 6

Hub locations in optimal solutions for the Turkey data.

\begin{tabular}{lllll}
\hline$p$ & $r=3$ & $r=4$ & $r=5$ & $r=p$ \\
\hline 5 & $1,3,6,23,34$ & $1,6,23,34,35$ & $1,6,23,34,35$ & $1,6,23,34,35$ \\
6 & $1,3,6,23,34$, & $1,6,21,34,35$, & $1,6,21,34,35$, & $1,6,21,34,35$, \\
& 55 & 55 & 55 & 55 \\
7 & 1,3, & 1,3, & 1,3, & 1,3, \\
& $6,23,34,35$, & $6,21,34,35,55$ & $6,21,34,35,55$ & $6,21,34,35,55$ \\
& 55 & & & \\
8 & $1,3,6$ & $1,3,6$, & $1,3,6$, & $1,3,6$, \\
& $25,34,35,44$, & $21,25,34,35$, & $21,25,34,35$, & $21,25,34,35$, \\
& 55 & 38 & 38 & 55 \\
9 & & $1,3,6,21$, & $1,3,6,21$, & $1,3,6,21$, \\
& & $25,34,35,38$, & $25,34,35,38$, & $25,34,35,38$, \\
& & 55 & 55 & 55 \\
10 & $1,3,6,16,21$, & $1,3,6,16,21$, & $1,3,6,16,21$, & $1,3,6,16,21$, \\
& $25,34,35,38$, & $25,34,35,38$, & $25,34,35,38$, & $25,34,35,38$, \\
& 55 & 55 & 55 & 55 \\
\hline
\end{tabular}


For $p=4$, the hub locations are the same for $\alpha=0.6,0.4,0.2$ with one exception; the hub located at node 1 (Atlanta) moves to node 24 (Tampa) as $r$ increases from 1 to 2 for $\alpha=0.4$. For $\alpha=1$, node 4 is the only common hub node for different values of $r$. Hubs are centrally located, at nodes 4, 7 (Dallas), 8, and 20, in the single allocation solution. Two hubs move towards the east and west coasts, to nodes 18 and 12 for $r=2$. As $r$ increases further, there is a slight change, the hub at node 13 (Memphis) is replaced with 1 . For $\alpha=0.8$, nodes 4 and 12 are among the hub nodes for all values of $r$. In the single allocation solution, the other two hubs are located at nodes 1 and 18 . These move to nodes 13 and 17 when $r=2$. When $r$ increases to 3 and 4 , the hub at node 13 moves back to node 1 .

For the CAB instances with $p=5$ and $\alpha=1$, nodes 1,4 , and 7 appear as hubs for all values of $r$ considered. The hub at node 2 in the single allocation solution moves to node 18 , and then to node 17 as $r$ increases. These nodes are located in the same region. However, the other hub at node 8 , which is located more in the center in the single allocation solution, moves to node 12 , on the west coast of the United States, for $r=2,3, p$. Hubs are located at nodes 4, 7, and 12 in all the solutions for $p=5$ and $\alpha=0.8$. Other than these, a hub is located at node 1 for $r=1,2,3$ and it moves south-east to node 24 in the multiple allocation solution. The fifth hub, located at node 18 for $r=1$, moves to node 17 for larger $r$ values. Finally for $\alpha=0.6$, four hubs are located at nodes $4,7,12$, and 17 in all solutions. The fifth hub is located at node 14 (Miami) in the single allocation solution, moves to node 24 for $r=2,3$, and then moves back to node 14 in the multiple allocation solution. Nodes 14 and 24 are located in the south-east and are close to each other. For smaller $\alpha$ values, the locations of hubs are not affected by the allocation strategy. This is expected since nodes are often allocated to the closest hubs when $\alpha$ is small, as discussed in the Introduction.

For the AP instances, we observe that hub locations remain almost the same for all allocation strategies; only slight changes occur for $r=1$ and 2 .

Finally, we analyze the solutions for the Turkish data. We refer the reader to the map given in Fig. 5 in Yaman (2009). We note here that the problem with $p=9$ and $r=3$ could not be solved to optimality, hence, we cannot report the optimal hub locations for this instance. Here we see that for $p=9,10$, hub locations are not affected by the choice of $r$. For smaller $p$ values, there are slight changes. For $p=5$, the four hubs are located at nodes 1 (Adana), 6 (Ankara), 23 (Elazig), and 34 (Istanbul) in all solutions. The fifth hub is located at node 3 (Afyon) for $r=3$ and moves west to node 35 (Izmir) for larger $r$ values. For $p=6$, again, four hubs are located at common nodes, namely, nodes $1,6,34$, and 55 (Samsun). For $r=3$, the other two hubs are located at nodes 3 and 23. For larger $r$, the hub at node 3 moves west to node 35 and the hub at node 23 moves slightly south-east to node 21 (Diyarbakir). For $p=7$, hubs are located at nodes $1,3,6,34,35$, and 55 for all $r$ values. The seventh hub is located at node 23 for $r=3$ and moves to node 21 as we increase $r$. Finally, for $p=8$, hubs are located at nodes $1,3,6,25$ (Erzurum), 34, and 35 for all $r$ values. For $r=3$, the remaining two hubs are located at nodes 44 (Malatya) and 55. The hub at node 44 moves south-east to node 21 and the hub at node 55 moves south towards the center of the country to node 38 (Kayseri). For $r=p$, the hub at node 38 moves back to 55 .

Overall, we can conclude that the allocation strategy does not have a large effect on hub locations in the optimal solutions for our instances. Most hub locations remain the same for different $r$ values. For those hubs that change location, we observe a slight movement away from the center as $r$ increases.

\subsection{Computation times}

In our last experiment, we investigate the computational performance of our formulations. We consider a third formulation which is obtained by aggregating constraints (23) in UrApHMP2 (see Ernst and Krishnamoorthy, 1998b). This new model, called UrApHMP3, is the same as UrApHMP2 except that constraints (23) are replaced with

$$
\sum_{i \in N} x_{l j}^{i} \leqslant \sum_{i \in N} t_{i j} z_{j l} \quad \forall j, l \in N .
$$

With this aggregation, the number of constraints decrease from $O\left(|N|^{3}\right)$ to $O\left(|N|^{2}\right)$, but the formulation becomes weaker.

We limit this experiment to datasets $\mathrm{CAB}$ with $p=5$ and AP with $n=50$. We are able to solve the CAB instances using all three formulations. However, with UrApHMP1, we ran out of memory in the AP instances, hence, we only report the results for formulations UrApHMP2 and UrApHMP3. For each instance and formulation, we report the linear programming relaxation gap, i.e., the percentage difference between the optimal value of the integer problem and the optimal value of its linear programming relaxation, in column 'gap', the cpu time in seconds in column 'cpu', and the number of nodes evaluated in the branch-and-cut tree in column 'nodes'. The best results are in boldface.

All instances are solved using GAMS 22.5 and CPLEX 11.0.0 on an AMD Opteron 252 processor $(2.6 \mathrm{GHz}$ ) with $2 \mathrm{~GB}$ of RAM operating under the system CentOS (Linux version 2.6.9-42.0.3.ELsmp).

In Table 7, we report the results for the CAB instances with $n=25, p=5, \alpha=1,0.8,0.6,0.4,0.2$, and $r=1,2,3,5$. Here we observe that UrApHMP1 is stronger than UrApHMP2, which is, in turn, stronger than UrApHMP3. The differences between gaps are quite large for difficult instances. For the instance with $r=1$ and $\alpha=1$, these gaps are $0.20 \%, 5.12 \%$, and $21.27 \%$, respectively. The differences are smaller for the instances with larger $r$ values and smaller $\alpha$ values. But even in these cases, UrApHMP3 is quite weak. For all instances, UrApHMP1 is the best in terms of number of nodes in the branch-and-cut tree. This formulation is also the best in terms of cpu time for all instances except those with $\alpha=0.2$. These latter instances are rather easy to solve; they are solved in a few seconds with all three formulations. However, for the difficult instances, i.e., the instances with large $\alpha$ and small $r$ values, UrApHMP1 has much shorter cpu times compared to the other two formulations. For the instance with $r=2$ and $\alpha=1$, the solution time is 225.41 with UrApHMP1, whereas it is 10059.71 and 14241.13 with UrApHMP2 and UrApHMP3, i.e., around 45 and 63 times larger, respectively. We can conclude that for the CAB data with 25 nodes, the largest formulation, UrApHMP1, clearly outperforms smaller but weaker formulations UrApHMP2 and UrApHMP3.

As mentioned above, the results for the AP instances are given in Table 8 and we can only compare UrApHMP2 and UrApHMP3 because we had memory problems with UrApHMP1. In these results, we see that UrApHMP2 outperforms UrApHMP3 in all instances and for all three measures. The differences between gaps are quite large in all instances. The largest gap with UrApHMP2 is less than $1 \%$, whereas the smallest gap with UrApHMP3 is 3.9\%. The average gaps are $0.55 \%$ and $4.86 \%$, for UrApHMP2 and UrApHMP3, respectively. The averages of the number of nodes are 35.33 and 94.83 . The difference is larger for the cpu times. The average cpu time with UrApHMP3 is 3313.51 seconds; this amounts to 3.67 times the average cpu time with UrApHMP2.

To summarize, when we analyze these results, we see that, as long as the memory permits, stronger formulations perform better despite their large sizes.

\section{Conclusion}

In this paper, we introduce a new problem, called the uncapacitated $r$-allocation $p$-hub median problem. Here, each node can be allocated to at most $r$ nodes. This problem generalizes the single 
Table 7

Comparison of formulations for the CAB data with $n=25$ and $p=5$.

\begin{tabular}{|c|c|c|c|c|c|c|c|c|c|c|c|}
\hline \multirow[t]{2}{*}{$r$} & \multirow[t]{2}{*}{$\alpha$} & \multirow[t]{2}{*}{ Optimal } & \multicolumn{3}{|c|}{ UrApHMP1 } & \multicolumn{3}{|c|}{ UrApHMP2 } & \multicolumn{3}{|c|}{ UrApHMP3 } \\
\hline & & & Gap & cpu & Nodes & Gap & cpu & Nodes & Gap & cpu & Nodes \\
\hline \multirow[t]{6}{*}{1} & 1 & 1173.24 & 0.20 & 82.34 & 7 & 5.12 & 1064.86 & 5080 & 21.27 & 11508.90 & 3620 \\
\hline & 0.8 & 1034.10 & 0.00 & 22.60 & $\mathbf{0}$ & 4.48 & 338.52 & 1067 & 17.15 & 9292.36 & 981 \\
\hline & 0.6 & 876.59 & 0.00 & 19.06 & $\mathbf{0}$ & 3.48 & 99.45 & 250 & 12.63 & 2178.39 & 407 \\
\hline & 0.4 & 707.69 & 0.00 & 7.75 & $\mathbf{0}$ & 1.49 & 19.00 & 20 & 7.44 & 76.78 & 46 \\
\hline & 0.2 & 538.39 & 0.00 & 4.85 & $\mathbf{0}$ & 0.23 & 4.29 & 3 & 2.53 & 5.92 & 7 \\
\hline & & Average & 0.04 & 27.32 & 1.40 & 2.96 & 305.22 & 1284.00 & 12.20 & 4612.47 & 1012.20 \\
\hline \multirow[t]{6}{*}{2} & 1 & 1018.75 & 0.91 & 225.41 & 76 & 2.16 & 10059.71 & 3827 & 9.33 & 14241.13 & 3273 \\
\hline & 0.8 & 934.90 & 0.62 & 94.32 & 37 & 2.14 & 1589.40 & 1732 & 8.34 & 3554.24 & 1230 \\
\hline & 0.6 & 818.56 & 0.00 & 12.52 & $\mathbf{0}$ & 1.56 & 90.26 & 326 & 6.43 & 256.75 & 362 \\
\hline & 0.4 & 681.81 & 0.00 & 4.70 & $\mathbf{0}$ & 0.51 & 18.46 & 32 & 3.92 & 42.14 & 36 \\
\hline & 0.2 & 530.34 & 0.00 & 3.75 & $\mathbf{0}$ & 0.01 & 1.81 & $\mathbf{0}$ & 1.04 & 4.96 & $\mathbf{0}$ \\
\hline & & Average & 0.31 & 68.14 & 22.60 & 1.28 & 2351.93 & 1183.40 & 5.81 & 3619.84 & 980.20 \\
\hline \multirow[t]{6}{*}{3} & 1 & 981.58 & 0.00 & 8.75 & $\mathbf{0}$ & 0.24 & 169.78 & 87 & 5.90 & 595.58 & 158 \\
\hline & 0.8 & 912.23 & 0.03 & 22.13 & 3 & 0.46 & 60.96 & 89 & 6.08 & 60.10 & 46 \\
\hline & 0.6 & 807.47 & 0.00 & 5.74 & $\mathbf{0}$ & 0.63 & 33.11 & 69 & 5.16 & 62.68 & 92 \\
\hline & 0.4 & 678.03 & 0.00 & 4.80 & $\mathbf{0}$ & 0.20 & 11.12 & 8 & 3.40 & 25.56 & 19 \\
\hline & 0.2 & 530.00 & 0.00 & 3.74 & $\mathbf{0}$ & 0.00 & 1.32 & $\mathbf{0}$ & 0.99 & 3.43 & $\mathbf{0}$ \\
\hline & & Average & 0.01 & 9.03 & 0.60 & 0.30 & 55.26 & 50.60 & 4.31 & 149.47 & 63.00 \\
\hline \multirow[t]{6}{*}{5} & 1 & 975.24 & 0.00 & 5.18 & $\mathbf{0}$ & 0.00 & 5.68 & $\mathbf{0}$ & 5.29 & 25.69 & 0 \\
\hline & 0.8 & 910.35 & 0.00 & 6.15 & $\mathbf{0}$ & 0.32 & 30.86 & 20 & 5.85 & 52.71 & 46 \\
\hline & 0.6 & 804.70 & 0.00 & 5.48 & $\mathbf{0}$ & 0.35 & 24.08 & 25 & 4.82 & 44.50 & 56 \\
\hline & 0.4 & 676.34 & 0.00 & 5.23 & $\mathbf{0}$ & 0.03 & 7.80 & $\mathbf{0}$ & 3.14 & 18.00 & $\mathbf{0}$ \\
\hline & 0.2 & 530.00 & 0.00 & 4.19 & $\mathbf{0}$ & 0.00 & 1.76 & $\mathbf{0}$ & 0.99 & 3.26 & $\mathbf{0}$ \\
\hline & & Average & 0.00 & 5.25 & 0.00 & 0.14 & 14.04 & 9.00 & 4.02 & 28.83 & 20.40 \\
\hline
\end{tabular}

Table 8

Comparison of formulations for the AP data with $n=50$.

\begin{tabular}{|c|c|c|c|c|c|c|c|c|}
\hline \multirow[t]{2}{*}{$r$} & \multirow[t]{2}{*}{$p$} & \multirow[t]{2}{*}{ Optimal } & \multicolumn{3}{|c|}{ UrApHMP2 } & \multicolumn{3}{|c|}{ UrApHMP3 } \\
\hline & & & Gap & $\mathrm{cpu}$ & Nodes & Gap & $\mathrm{cpu}$ & Nodes \\
\hline \multirow[t]{3}{*}{1} & 3 & 158569.93 & 0.49 & 1038.43 & 32 & 5.92 & 7072.40 & 161 \\
\hline & 4 & 143378.05 & 0.62 & 1189.92 & 35 & 6.11 & 8565.98 & 179 \\
\hline & 5 & 132366.95 & 0.95 & 1047.97 & 91 & 5.93 & 6012.50 & 191 \\
\hline \multirow[t]{3}{*}{2} & 3 & 156160.77 & 0.29 & 791.03 & 11 & 4.66 & 3243.53 & 25 \\
\hline & 4 & 141405.48 & 0.58 & 1440.95 & 30 & 4.93 & 2302.48 & 100 \\
\hline & 5 & 129773.61 & 0.75 & 1081.30 & 82 & 4.17 & 1772.34 & 112 \\
\hline \multirow[t]{3}{*}{3} & 3 & 156014.73 & 0.24 & 600.19 & 7 & 4.57 & 2742.73 & 24 \\
\hline & 4 & 141258.07 & 0.56 & 863.98 & 30 & 4.83 & 1772.14 & 98 \\
\hline & 5 & 129513.80 & 0.72 & 769.27 & 44 & 3.97 & 1049.42 & 89 \\
\hline \multirow[t]{3}{*}{$p$} & 3 & 156014.73 & 0.24 & 580.43 & 7 & 4.57 & 2677.03 & 24 \\
\hline & 4 & 141153.38 & 0.50 & 683.18 & 11 & 4.76 & 1661.99 & 86 \\
\hline & 5 & 129412.60 & 0.67 & 735.54 & 44 & 3.90 & 889.55 & 49 \\
\hline
\end{tabular}

and multiple allocation versions of the $p$-hub median problem. We propose two mixed-integer programming formulations for this problem. We show that these formulations boil down to the well-known four-index and three-index formulations for the single and multiple allocation $p$-hub median problem when $r=1$ and $r=p$, respectively. We discuss several variations of the problem and propose ways to model these variations. We underline the difference between imposing upper bounds on path lengths and delivery times for $r \geqslant 2$. Finally, we perform a computational study and discuss the outcomes.

The results of our computational study lead us to the following conclusions: We first conclude that single allocation solutions may be much more expensive than multiple allocation solutions, however, allowing a node to be allocated to one or two more hubs may result in considerable savings in terms of the routing cost. The resulting solutions are likely to be cheaper than multiple allocation solutions when the fixed costs related to the nonhub-hub connec- tions are also taken into account. Moreover, the resulting networks are likely to be much easier to operate and manage because they have fewer connections compared to multiple allocation networks.

Second, we conclude that the allocation strategies do not have a large impact on hub locations in optimal solutions. This implies that companies may change their allocation strategies without a big need to move their hubs.

Finally, we observe that a formulation's strength is important in solving UrApHMP instances. Hence, an interesting future research direction would be to derive stronger formulations for this problem.

\section{Acknowledgements}

We are grateful to two referees for their comments and suggestions that improved the content. This research is supported by TUBITAK Grant No. 107M460. 


\section{References}

Abdinnour-Helm, S., 1998. A hybrid heuristic for the uncapacitated hub location problem. European Journal of Operational Research 106, 489-499.

Abdinnour-Helm, S., 2001. Using simulated annealing to solve the $p$-hub median problem. International Journal of Physical Distribution and Logistics Management 31, 203-220.

Abdinnour-Helm, S., Venkataramanan, M.A., 1998. Solution approaches to hub location problems. Annals of Operations Research 78, 31-50.

Alumur, S., Kara, B.Y., 2008. Network hub location problems: The state of the art European Journal of Operational Research 190, 1-21.

Alumur, S., Kara, B.Y., Karasan, O.E., 2009. The design of single allocation incomplete hub networks. Transportation Research Part B 43, 936-951.

Aykin, T., 1994. Lagrangian relaxation based approaches to capacitated hub-andspoke network design problem. European Journal of Operational Research 79, 501-523.

Aykin, T., 1995. Networking policies for hub-and-spoke systems with application to the air transportation system. Transportation Science 29, 201-221.

Boland, N., Krishnamoorthy, M., Ernst, A.T., Ebery, J., 2004. Preprocessing and cutting for multiple allocation hub location problems. European Journal of Operational Research 155, 638-653.

Calik, H., Alumur, S., Kara, B.Y., Karasan, O.E., 2009. A tabu-search based heuristic for the hub covering problem over incomplete hub networks. Computers and Operations Research 36, 3088-3096.

Camargo, R.S., Miranda Jr., G., Luna, H.P., 2008. Benders decomposition for the uncapacitated multiple allocation hub location problem. Computers and Operations Research 35, 1047-1064.

Campbell, J.F., 1992. Location and allocation for distribution systems with transshipments and transportation economies of scale. Annals of Operations Research 40, 77-99.

Campbell, J.F., 1994. Integer programming formulations of discrete hub location problems. European Journal of Operational Research 72, 387-405.

Campbell, J.F., 1996. Hub location and the $p$-hub median problem. Operations Research 44, 923-935.

Campbell, J.F., 2009. Hub location for time definite transportation. Computers and Operations Research 36, 3107-3116.

Campbell, J.F., Ernst, A.T., Krishnamoorthy, M., 2002. Hub location problems. In: Drezner, Z., Hamacher, H. (Eds.), Facility Location: Applications and Theory. Springer-Verlag, Berlin, pp. 373-407.

Campbell, J.F., Ernst, A.T., Krishnamoorthy, M., 2005a. Hub arc location problems: Part I - Introduction and results. Management Science 51, 1540-1555.

Campbell, J.F., Ernst, A.T., Krishnamoorthy, M., 2005b. Hub arc location problems: Part II - Formulations and optimal algorithms. Management Science 51, 15561571.

Cánovas, L., García, S., Marín, A., 2007. Solving the uncapacitated multiple allocation hub location problem by means of a dual-ascent technique. European Journal of Operational Research 179, 990-1007.

Chen, J.F., 2007. A hybrid heuristic for the uncapacitated single allocation hub location problem. Omega 35, 211-220.

Contreras, I., Fernández, E., Marín, A., 2010. The tree of hubs location problem. European Journal of Operational Research 202, 390-400.

Cunha, C.B., Silva, M.R., 2007. A genetic algorithm for the problem of configuring a hub-and-spoke network for a LTL trucking company in Brazil. European Journal of Operational Research 179, 747-758.

Ebery, J., 2001. Solving large single allocation $p$-hub problems with two or three hubs. European Journal of Operational Research 128, 447-458.

Ernst, A.T., Krishnamoorthy, M., 1996. Efficient algorithms for the uncapacitated single allocation $p$-hub median problem. Location Science 4, 139-154.

Ernst, A.T., Krishnamoorthy, M., 1998a. An exact solution approach based on shortest paths for $p$-hub median problems. INFORMS Journal on Computing 10 , 149-162.

Ernst, A.T., Krishnamoorthy, M., 1998b. Exact and heuristic algorithms for the uncapacitated multiple allocation $p$-hub median problems. European Journal of Operational Research 104, 100-112.

Ernst, A.T., Hamacher, H.W., Jiang, H., Krishnamoorthy, M., Woeginger, G., 2009. Uncapacitated single and multi allocation $p$-hub center problems. Computers and Operations Research 36, 2230-2241.

Hamacher, H.W., Labbé, M., Nickel, S., Sonneborn, T., 2004. Adapting polyhedral properties from facility to hub location problems. Discrete Applied Mathematics 145, 104-116.

Horner, M.W., O'Kelly, M.E., 2001. Embedding economies of scale concepts for hub network design. Journal of Transport Geography 9, 255-265.

Ilić, A., Urošević, D., Brimberg, J., Mladenović, N., 2010. A general variable neighborhood search for solving the uncapacitated single allocation $p$-hub median problem. European Journal of Operational Research 206, 289-300.

Kara, B.Y., Tansel, B.C., 2000. On the single-assignment $p$-hub center problem. European Journal of Operational Research 125, 648-655.

Kara, B.Y., Tansel, B.C., 2001. The latest arrival hub location problem. Management Science $47,1408-1420$.

Klincewicz, J.G., 1991. Heuristics for the $p$-hub location problem. European Journal of Operational Research 53, 25-37.
Klincewicz, J.G., 1992. Avoiding local optima in the $p$-hub location problem using tabu search and GRASP. Annals of Operations Research 40, 283-302.

Klincewicz, J.G., 1996. A dual algorithm for the uncapacitated hub location problem. Location Science 4, 173-184.

Kratica, J., Stanimirović, Z., Tosić, D., Filipović, V., 2007. Two genetic algorithms for solving the uncapacitated single allocation $p$-hub median problem. European Journal of Operational Research 182, 15-28.

Labbé, M., Yaman, H., 2004. Projecting flow variables for hub location problems. Networks 44, 84-93.

Labbé, M., Yaman, H., 2008. Solving the hub location problem in a star-star network. Networks 51, 19-33.

Marín, A., 2005. Uncapacitated Euclidean hub location: Strengthened formulation, new facets and a relax-and-cut algorithm. Journal of Global Optimization 33, 393-422.

Marín, A., Cánovas, L., Landete, M., 2006. New formulations for the uncapacitated multiple allocation hub location problem. European Journal of Operational Research 172, 274-292.

Mayer, G., Wagner, B., 2002. HubLocator: An exact solution method for the multiple allocation hub location problem. Computers and Operations Research 29, 715739.

Nickel, S., Schobel, A., Sonneborn, T., 2001. Hub location problems in urban traffic networks. In: Niittymaki, J., Pursula, M. (Eds.), Mathematics Methods and Optimization in Transportation Systems. Kluwer, pp. 1-12.

O'Kelly, M.E., 1987. A quadratic integer program for the location of interacting hub facilities. European Journal of Operational Research 32, 393-404.

O'Kelly, M.E., 1992. Hub facility location with fixed costs. Papers in Regional Science 71, 293-306.

O'Kelly, M.E., Bryan, D., 1998. Hub location with flow economies of scale. Transportation Research Part B 32, 605-616.

O'Kelly, M.E., Miller, H.J., 1991. Solution strategies for the single facility minimax hub location problem. Papers in Regional Science 70, 367-380.

O'Kelly, M.E., Miller, H.J., 1994. The hub network design problem: A review and synthesis. Journal of Transport Geography 2, 31-40.

O'Kelly, M.E., Skorin-Kapov, D., Skorin-Kapov, J., 1995. Lower bounds for the hub location problem. Management Science 41, 713-721.

O’Kelly, M.E., Bryan, D., Skorin-Kapov, D., Skorin-Kapov, J., 1996. Hub network design with single and multiple allocation: A computational study. Location Science 4, 125-138

Pirkul, H., Schilling, D., 1998. An efficient procedure for designing single allocation hub and spoke systems. Management Science 44, S235-S242.

Podnar, H., Skorin-Kapov, J., Skorin-Kapov, D., 2002. Network cost minimization using threshold-based discounting. European Journal of Operational Research 137, 371-386

Silva, M.R., Cunha, C.B., 2009. New simple and efficient heuristics for the uncapacitated single allocation hub location problem. Computers and Operations Research 36, 3152-3165.

Skorin-Kapov, D., Skorin-Kapov, J., 1994. On tabu search for the solution of interacting hub facilities. European Journal of Operational Research 73, 502509.

Skorin-Kapov, D., Skorin-Kapov, J., O’Kelly, M.E., 1996. Tight linear programming relaxations of uncapacitated $p$-hub median problems. European Journal of Operational Research 94, 582-593.

Smith, K., Krishnamoorthy, M., Palaniswami, M., 1996. Neural versus traditional approaches to the location of interacting hub facilities. Location Science 4, 155171.

Sohn, J., Park, S., 1997. A linear program for the two hub location problem. European Journal of Operational Research 100, 617-622.

Sohn, J., Park, S., 1998. Efficient solution procedure and reduced size formulations for $p$-hub location problems. European Journal of Operational Research 108, $118-126$.

Sung, C.S., Jin, H.W., 2001. Dual-based approach for a hub network design problem under non-restrictive policy. European Journal of Operational Research 132, 88105.

Topcuoglu, H., Corut, F., Ermis, M., Yilmaz, G., 2005. Solving the uncapacitated hub location problem using genetic algorithms. Computers and Operations Research 32, 967-984.

Wagner, B., 2004. A note on the latest arrival hub location problem. Management Science 50, 1751-1755.

Wagner, B., 2007. An exact solution procedure for a cluster hub location problem. European Journal of Operational Research 178, 391-401.

Yaman, H., 2008. Star p-hub median problem with modular arc capacities. Computers and Operations Research 35, 3009-3019.

Yaman, H., 2009. The hierarchical hub median problem with single assignment. Transportation Research Part B 43, 643-658.

Yaman, H., Kara, B.Y., Tansel, B.C., 2007. The latest arrival hub location problem for cargo delivery systems with stopovers. Transportation Research Part B 41, 906919 .

Yoon, M.G., Current, J., 2008. The hub location and network design problem with fixed and variable arc costs: Formulation and dual-based solution heuristic. Journal of the Operational Research Society 59, 80-89. 